\title{
An example for nonexistence of minimal foliations
}

Xue-Qing Miao ${ }^{1 *}$ (D)

\section{"Correspondence:} ntitmxq@163.com

'school of Sciences, Nantong University, Nantong, 226019, China

\begin{abstract}
For the high-dimensional Frenkel-Kontorova model on lattices, we have concluded that there are heteroclinic connections between neighboring Birkhoff minimizers which are more periodic. This conclusion is based on the existence of neighboring elements, i.e., the existence of gaps. By adding a large enough oscillation to the local potential, I prove that all minimal foliations can be destroyed into minimal laminations, and hence there always exist gaps.
\end{abstract}

MSC: 37E40; 37J50; 37K60; 82C22

Keywords: Aubry-Mather theory; High-dimensional Frenkel-Kontorova model; Minimal foliation; Minimal lamination

\section{Introduction}

The Aubry-Mather theory for the classical Frenkel-Kontorova (FK) model [1] or for monotone twist maps [14] has been extended to high-dimensional lattice systems [7, 8, $10,12,15,17,19]$ and to PDEs [2, 5, 6, 9, 16]. In this paper, we study the high-dimensional FK model on lattices. Let $B \subset \mathbb{Z}^{d}$ be a finite set containing the origin, which is called the basic neighborhood of interactions, $h: \mathbb{R}^{B} \rightarrow \mathbb{R}$ be a $C^{2}$-smooth function satisfying some hypotheses specified in Sect. 2. Let $\tau_{k, l}\left(k \in \mathbb{Z}^{d}, l \in \mathbb{Z}\right)$ denote the $\mathbb{Z}^{d+1}$ translate on the configuration space $\mathbb{R}^{\mathbb{Z}^{d}}:\left(\tau_{k, l} \mathbf{x}\right)_{j}=x_{j-k}+l$ for all $j \in \mathbb{Z}^{d}$, in which $\mathbf{x}=\left(x_{j}\right) \in \mathbb{R}^{\mathbb{Z}^{d}}$. We denote $h_{j}(\mathbf{x})=h\left(\left.\tau_{-j, 0} \mathbf{x}\right|_{B}\right)$ and by $W(\mathbf{x})=\sum_{j \in \mathbb{Z}^{d}} h_{j}(\mathbf{x})$ the formal Lagrangian.

Like the classical FK model, we study minimizers for the local potential $h$. A configuration $\mathbf{x}$ is said to be a minimizer of $W$ if $W(\mathbf{x}+\mathbf{v})-W(\mathbf{x}) \geq 0$ for every compactly supported configuration $\mathbf{v} \in \mathbb{R}^{\mathbb{Z}^{d}}$. Unlike the classical FK model, for which all minimizers are Birkhoff $[1,4]$, minimizers for the high-dimensional FK model or even monotone recurrence relations may not be Birkhoff, see [18] for an example. A configuration $\mathbf{x}$ is said to be Birkhoff if the translation $\left\{\tau_{k, l} \mathbf{x} \mid k \in \mathbb{Z}^{d}, l \in \mathbb{Z}\right\}$ of $\mathbf{x}$ is totally ordered (see Sect. 2 for the definition). Each Birkhoff configuration on $\mathbb{Z}^{d}$ has a rotation vector which turns out to be the rotation number for $d=1$.

Let $\mathscr{M}$ denote the set of all Birkhoff minimizers and $\mathscr{M}_{\omega}$ denote the set of all Birkhoff minimizers with rotation vector $\omega \in \mathbb{R}^{d}$. Then the Aubry-Mather theory for the highdimensional FK model tells us that $\mathscr{M}_{\omega} \neq \emptyset$ (see, for example, $[7,8,17]$ ) if the local potential $h$ satisfies hypotheses $(\mathrm{H} 1)-(\mathrm{H} 3)$ specified in Sect. 2.

(c) The Author(s) 2021. This article is licensed under a Creative Commons Attribution 4.0 International License, which permits use sharing, adaptation, distribution and reproduction in any medium or format, as long as you give appropriate credit to the original author(s) and the source, provide a link to the Creative Commons licence, and indicate if changes were made. The images or other third party material in this article are included in the article's Creative Commons licence, unless indicated otherwise in a credit line to the material. If material is not included in the article's Creative Commons licence and your intended use is not permitted by statutory regulation or exceeds the permitted use, you will need to obtain permission directly from the copyright holder. To view a copy of this licence, visit http://creativecommons.org/licenses/by/4.0/. 
According to Moser [16], $\mathscr{M}_{\omega}$ is called a minimal foliation if $p_{0}\left(\mathscr{M}_{\omega}\right)=\mathbb{R}$ (see Sect. 3 for the definition), where $p_{0}$ is the projection on 0 - site, i.e., $p_{0}(\mathbf{x})=x_{0}$. Otherwise it is called a minimal lamination. For the classical FK model or monotone twist maps, minimal foliations correspond to invariant circles which play an important role in the discussion of monotone twist maps.

For the high-dimensional FK model, the rotation vector is not enough to distinguish different minimizers. Therefore we introduce secondary invariants of Birkhoff minimizers with the same rotation vector [15] as Bangert did in [5].

Assume that $\mathbf{x} \in \mathbb{R}^{\mathbb{Z}^{d}}$ is a Birkhoff configuration with rotation vector $\omega$. If $(k, l) \cdot(-\omega, 1)=$ 0 , we do not know whether $\tau_{k, l} \mathbf{x} \gg \mathbf{x}$ or $\tau_{k, l} \mathbf{x}=\mathbf{x}$ or $\tau_{k, l} \mathbf{x} \ll \mathbf{x}$. But we do know that the set $\bar{\Gamma}=\left\{(k, l) \in \mathbb{Z}^{d} \times \mathbb{Z} \mid(k, l) \cdot(-\omega, 1)=0\right\}$ is a subgroup of $\mathbb{Z}^{d+1}$ and the orbit of $\bar{\Gamma}$-action $\left\{\tau_{k, l} \mathbf{x} \mid(k, l) \in \bar{\Gamma}\right\}$ is totally ordered since $\mathbf{x}$ is Birkhoff. Then there exists an integer $t$ with $1 \leq t \leq d+1$ and unit vectors $\bar{a}_{1}, \ldots, \bar{a}_{t}$ with $\bar{a}_{s} \in \operatorname{span}\left(\mathbb{Z}^{d+1} \cap\left\langle\bar{a}_{1}, \ldots, \bar{a}_{s-1}\right\rangle^{\perp}\right)(1<s \leq t)$ such that $(k, l) \in \mathbb{Z}^{d+1} \cap\left\langle\bar{a}_{1}, \ldots, \bar{a}_{s-1}\right\rangle^{\perp}$ and $(k, l) \cdot \bar{a}_{s}>0$ imply $\tau_{k, l} \mathbf{x} \gg \mathbf{x}$, and $\tau_{k, l} \mathbf{x}=\mathbf{x}$ if $(k, l) \in \mathbb{Z}^{d+1} \cap\left\langle\bar{a}_{1}, \ldots, \bar{a}_{t}\right\rangle^{\perp}$, see [15] for details. Here, we just introduce the concepts we need in [15].

Definition 1.1 The integer $t=t(\mathbf{x})$ and the unit vectors $\bar{a}_{1}(\mathbf{x}), \ldots, \bar{a}_{t}(\mathbf{x})$ are said to be the secondary invariants of a Birkhoff configuration $\mathbf{x}$.

We remark that the integer $t$ and the unit vectors $\bar{a}_{1}, \ldots, \bar{a}_{t}$ depend upon $\mathbf{x}$. For notational convenience, we set $\bar{\Gamma}_{1}=\mathbb{Z}^{d+1}$ and

$$
\bar{\Gamma}_{s}=\bar{\Gamma}_{s}(\mathbf{x})=\mathbb{Z}^{d+1} \cap\left\langle\bar{a}_{1}(\mathbf{x}), \ldots, \bar{a}_{s-1}(\mathbf{x})\right\rangle^{\perp}, \quad(2 \leq s \leq t+1)
$$

Then

$$
\bar{a}_{s}(\mathbf{x}) \in \operatorname{span}\left(\bar{\Gamma}_{s}(\mathbf{x})\right) \quad(2 \leq s \leq t) .
$$

Definition 1.2 A system $\left(\bar{a}_{1}, \ldots, \bar{a}_{t}\right)$ of unit vectors in $\mathbb{R}^{d+1}$ is said to be admissible if $\bar{a}_{1}$. $\bar{e}_{d+1}>0$ and if, for $2 \leq s \leq t$,

$$
\bar{a}_{s} \in \operatorname{span}\left(\bar{\Gamma}_{s}\right), \quad \text { where } \bar{\Gamma}_{s}=\bar{\Gamma}_{s}\left(\bar{a}_{1}, \ldots, \bar{a}_{t}\right)=\mathbb{Z}^{d+1} \cap\left\langle\bar{a}_{1}, \ldots, \bar{a}_{s-1}\right\rangle^{\perp} .
$$

Here, $\bar{e}_{d+1}=(0, \ldots, 0,1) \in \mathbb{R}^{d+1}$.

Definition 1.3 Assume that $\left(\bar{a}_{1}, \ldots, \bar{a}_{t}\right)$ is admissible. Define

$$
\mathscr{M}\left(\bar{a}_{1}, \ldots, \bar{a}_{t}\right)=\left\{\mathbf{x} \in \mathscr{M} \mid t(\mathbf{x})=t \text { and } \bar{a}_{s}(\mathbf{x})=\bar{a}_{s} \text { for } 1 \leq s \leq t\right\} .
$$

We remark that if $\left(\bar{a}_{1}, \ldots, \bar{a}_{t}\right)$ is admissible, then $\left(\bar{a}_{1}, \ldots, \bar{a}_{s}\right)$ is admissible for $1 \leq s<t$. In [15], we have got an important conclusion: If $\mathbf{x}^{1} \ll \mathbf{x}^{2}$ are neighboring elements in $\mathscr{M}\left(\bar{a}_{1}, \ldots, \bar{a}_{t-1}\right)$, then there exists $\mathbf{x} \in \mathscr{M}\left(\bar{a}_{1}, \ldots, \bar{a}_{t}\right)$ such that $\mathbf{x}^{1} \ll \mathbf{x} \ll \mathbf{x}^{2}$ and $\mathbf{x}$ is a heteroclinic orbit connecting the neighboring elements in $\mathscr{M}\left(\bar{a}_{1}, \ldots, \bar{a}_{t-1}\right)$ (This is Theorem 10.1 in [15]). For the discussion of heteroclinic orbits, we can also refer to reference [13]. [13] gives a pure variational viewpoint of the heteroclinic orbits that is different from reference [15], but the conclusions of the two references are similar. 
Let $\mathbf{u}, \mathbf{v} \in \mathscr{M}\left(\bar{a}_{1}, \ldots, \bar{a}_{t}\right)$ and $\mathbf{u} \ll \mathbf{v}$. If there is no $\mathbf{x} \in \mathscr{M}\left(\bar{a}_{1}, \ldots, \bar{a}_{t}\right)$ such that $\mathbf{u} \ll \mathbf{x} \ll \mathbf{v}$, then $\mathbf{u}$ and $\mathbf{v}$ are neighboring elements in $\mathscr{M}\left(\bar{a}_{1}, \ldots, \bar{a}_{t}\right)$. If there is a pair of neighboring elements in $\mathscr{M}\left(\bar{a}_{1}, \ldots, \bar{a}_{t}\right)$, then $\mathscr{M}\left(\bar{a}_{1}, \ldots, \bar{a}_{t}\right)$ is said to have a gap.

But in [15] we did not answer the question: how to ensure the existence of neighboring elements i.e., the existence of gaps in $\mathscr{M}\left(\bar{a}_{1}, \ldots, \bar{a}_{t-1}\right)$ ? In this paper, by the anti-integrable limit method, we construct an example for which there exist gaps in $\mathscr{M}\left(\bar{a}_{1}, \ldots, \bar{a}_{t-1}\right)$, see [3] for a similar conclusion for elliptic PDEs. By adding a large enough oscillation to the local potential, one can prove by the implicit function theorem that all minimal foliations can be destroyed into minimal laminations (like Theorem 3.3 together with Theorem 5.4 in [11]) and hence there always exist gaps in $\mathscr{M}\left(\bar{a}_{1}, \ldots, \bar{a}_{t-1}\right)$.

For this purpose, we consider that the formal Lagrangian $W^{\varepsilon}: \mathbb{R}^{\mathbb{Z}^{d}} \rightarrow \mathbb{R}$ is defined by

$$
W^{\varepsilon}(\mathbf{x})=\sum_{j \in \mathbb{Z}^{d}} h_{j}^{\varepsilon}(\mathbf{x}), \quad \text { with } h_{j}^{\varepsilon}(\mathbf{x})=V\left(x_{j}\right)+\varepsilon h_{j}(\mathbf{x}) .
$$

Then we are interested in variational monotone lattice recurrence relations of the form

$$
V^{\prime}\left(x_{i}\right)+\varepsilon \sum_{j \in \mathbb{Z}^{d}} \partial_{i} h_{j}(\mathbf{x})=0, \quad i \in \mathbb{Z}^{d} \text { and } \mathbf{x}=\left(x_{i}\right)_{i \in \mathbb{Z}^{d}} \in \mathbb{R}^{\mathbb{Z}^{d}}
$$

Equation (1.3) describes a lattice of particles in periodic background that experience ferromagnetic attraction. The background potential $V: \mathbb{R} \rightarrow \mathbb{R}$ is a twice continuously differentiable and one-periodic Morse function. It is clear that equation (1.3) describes the stationary points of the formal Lagrangian $W^{\varepsilon}$. Our main result is the following.

Theorem A Assume that $\left(\bar{a}_{1}, \ldots, \bar{a}_{t}\right)$ is an admissible system. For a sufficiently small positive number $\varepsilon$, the minimal solutions set $\mathscr{M}\left(\bar{a}_{1}\right) \cup \cdots \cup \mathscr{M}\left(\bar{a}_{1}, \ldots, \bar{a}_{t-1}\right)$ corresponding to the local potential $h_{j}^{\varepsilon}\left(\right.$ or $\left.W^{\varepsilon}\right)$ is not a foliation.That is to say, there are gaps in $\mathscr{M}\left(\bar{a}_{1}, \ldots, \bar{a}_{t-1}\right)$.

\section{Preliminaries}

We consider the configuration space $\mathbb{R}^{\mathbb{Z}^{d}}=\left\{\mathbf{x}=\left(x_{k}\right) \mid \mathbf{x}: \mathbb{Z}^{d} \rightarrow \mathbb{R}\right\}$ with the topology of pointwise convergence. We define on the configuration space the relations $\leq,<$, and $\ll$ by $\mathbf{x} \leq \mathbf{y} \Leftrightarrow x_{k} \leq y_{k}$ for every $k \in \mathbb{Z}^{d}, \mathbf{x}<\mathbf{y} \Leftrightarrow \mathbf{x} \leq \mathbf{y}$ but $\mathbf{x} \neq \mathbf{y}, \mathbf{x} \ll \mathbf{y} \Leftrightarrow x_{k}<y_{k}$ for every $k \in \mathbb{Z}^{d}$, respectively. Similarly for $\geq$, >, and $\gg$. We say that $\mathbf{x}$ and $\mathbf{y}$ are totally ordered if $\mathbf{x} \ll \mathbf{y}$ or $\mathbf{x}=\mathbf{y}$ or $\mathbf{x} \gg \mathbf{y}$.

For $k=\left(k_{1}, \ldots, k_{d}\right) \in \mathbb{Z}^{d}$, define $\|k\|=\sum_{n=1}^{d}\left|k_{n}\right|$ and $B_{j}^{r}=\left\{k \in \mathbb{Z}^{d} \mid\|k-j\| \leq r\right\}, r \in \mathbb{N}$ and $j \in \mathbb{Z}^{d}$. For $(k, l) \in \mathbb{Z}^{d} \times \mathbb{Z}$, the $\mathbb{Z}^{d+1}$ translate $\tau_{k, l}: \mathbb{R}^{\mathbb{Z}^{d}} \rightarrow \mathbb{R}^{\mathbb{Z}^{d}}$ is defined by

$$
\left(\tau_{k, l} \mathbf{x}\right)_{j}=x_{j-k}+l, \quad j \in \mathbb{Z}^{d}
$$

Definition 2.1 A configuration $\mathbf{x}$ is said to be Birkhoff if $\left\{\tau_{k, l} \mathbf{x} \mid k \in \mathbb{Z}^{d}, l \in \mathbb{Z}\right\}$ is totally ordered, i.e., for all $k \in \mathbb{Z}^{d}$ and $l \in \mathbb{Z}$, it follows that

$$
\tau_{k, l} \mathbf{x} \ll \mathbf{x} \quad \text { or } \quad \tau_{k, l} \mathbf{x}=\mathbf{x} \quad \text { or } \quad \tau_{k, l} \mathbf{x} \gg \mathbf{x} .
$$

Each Birkhoff configuration $\mathbf{x}=\left(x_{k}\right)$ has a rotation vector $\omega$ such that $\left\{x_{k}-k \cdot \omega\right\}$ in uniformly bounded [17]. Let $\mathcal{B}_{\omega}$ denote the set of Birkhoff configurations with rotation 
vector $\omega$. Then $\mathcal{B}_{\omega}$ is closed in the topology of pointwise convergence (product topology). Moreover, the rotation vector $\omega(\mathbf{x})$ depends continuously on $\mathbf{x}$ [17]. In other words, when $\mathbf{x}^{n} \in \mathcal{B}_{\omega_{n}}$ are Birkhoff configurations so that $\lim _{n \rightarrow \infty} \mathbf{x}^{n}=\mathbf{x}$ pointwise, then the limit $\lim _{n \rightarrow \infty} \omega_{n}=\omega$ exists and $\mathbf{x} \in \mathcal{B}_{\omega}$.

In this paper we fix for simplicity the basic neighborhood $B$ of interactions introduced in Sect. 1 to be $B_{0}^{r}$ in which $r \in \mathbb{N}$ is called the range of interactions. We assume that the $C^{2}$ local potential function $h: \mathbb{R}^{B_{0}^{r}} \rightarrow \mathbb{R}$ satisfies the following hypotheses:

(H1) $h(\xi+\tilde{\mathbf{1}})=h(\xi)$, where $\tilde{\mathbf{1}} \in \mathbb{R}^{B_{0}^{r}}$ with each component being 1 ;

$(\mathrm{H} 2) h$ is bounded from below and coercive in the following sense:

$$
\lim _{\left|\xi_{k}-\xi_{j}\right| \rightarrow \infty} h(\xi)=\infty \quad \text { for } k, j \in B_{0}^{r} \text { with }\|k-j\|=1
$$

(H3) $\partial_{i, k} h \leq 0$ for $i, k \in B_{0}^{r}$ with $i \neq k$, while $\partial_{0, k} h<0$ for $\|k\|=1$.

Let $A \subset \mathbb{Z}^{d}$ be a finite set. We call $\operatorname{int}(A)=\left\{j \in A \mid B_{j}^{r} \subset A\right\}$ the interior of $A, \bar{A}=\bigcup_{j \in A} B_{j}^{r}$ the closure of $A$, and $\partial A=\bar{A} \backslash \operatorname{int}(A)$ the boundary of $A$. Let

$$
W_{A}(\mathbf{x})=\sum_{j \in A} h_{j}(\mathbf{x}) \text { and } W(\mathbf{x})=\sum_{j \in \mathbb{Z}^{d}} h_{j}(\mathbf{x})
$$

where $h_{j}(\mathbf{x})=h\left(\left.\tau_{-j, 0} \mathbf{x}\right|_{B_{0}^{r}}\right)$. We remark that (H3) is called the twist condition, which is frequently used in the following form: For $\mathbf{x} \in \mathbb{R}^{\mathbb{Z}^{d}}$,

$$
\partial_{i, k} h_{j}(\mathbf{x}) \leq 0 \quad \text { for } i, k \in B_{j}^{r} \text { with } i \neq k \quad \text { and } \quad \partial_{j, k} h_{j}(\mathbf{x})<0 \quad \text { for }\|j-k\|=1 .
$$

We denote the support of a configuration $\mathbf{v}=\left(v_{j}\right)$ by $\operatorname{supp}(\mathbf{v})=\left\{j \in \mathbb{Z}^{d} \mid v_{j} \neq 0\right\}$. Let $V_{A}=$ $\{\mathbf{v} \mid \operatorname{supp}(\mathbf{v}) \subset \operatorname{int}(A)\}$.

Definition 2.2 A configuration $\mathbf{x}$ is called a minimizer for the local potential $h$ (or for the Lagrangian $W$ ) if, for every finite subset $A \subset \mathbb{Z}^{d}$ and every $\mathbf{v} \in V_{A}$,

$$
W_{A}(\mathbf{x}+\mathbf{v})-W_{A}(\mathbf{x}) \geq 0 .
$$

We remark that the limit of a sequence of minimizers is also a minimizer, and each minimizer automatically satisfies the following equation:

$$
\sum_{\|j-k\| \leq r} \partial_{k} h_{j}(\mathbf{x})=0 \quad \text { for all } k \in \mathbb{Z}^{d}
$$

For the following needs, we restate Theorem 10.1 in reference [15].

Theorem 2.3 (See Theorem 10.1 in [15]) Let $\left(\bar{a}_{1}, \ldots, \bar{a}_{t}\right)$ be an admissible system with $t \geq$ 2. If $\mathbf{x}^{1} \ll \mathbf{x}^{2}$ are neighboring elements in $\mathscr{M}\left(\bar{a}_{1}, \ldots, \bar{a}_{t-1}\right)$, then there exists $\mathbf{x} \in \mathscr{M}\left(\bar{a}_{1}, \ldots, \bar{a}_{t}\right)$ such that $\mathbf{x}^{1} \ll \mathbf{x} \ll \mathbf{x}^{2}$, and hence $\mathbf{x}^{-}=\mathbf{x}^{1}$ and $\mathbf{x}^{+}=\mathbf{x}^{2}$.

For $\mathbf{x} \in \mathscr{M}\left(\bar{a}_{1}, \ldots, \bar{a}_{t}\right)$, we always denote by $\mathbf{x}^{-}$and $\mathbf{x}^{+}$the elements in $\mathscr{M}\left(\bar{a}_{1}, \ldots, \bar{a}_{t-1}\right)$ defined by $\mathbf{x}^{-}=\lim _{n \rightarrow \infty} \tau_{k_{n}, l_{n}} \mathbf{x}$ for every sequence $\left(k_{n}, l_{n}\right)$ in $\bar{\Gamma}_{t}$ with $\lim _{n \rightarrow \infty}\left(k_{n}, l_{n}\right) \cdot \bar{a}_{t}=$ $-\infty$ and $\mathbf{x}^{+}=\lim _{n \rightarrow \infty} \tau_{k_{n}, l_{n}} \mathbf{x}$ for every sequence $\left(k_{n}, l_{n}\right)$ in $\bar{\Gamma}_{t}$ with $\lim _{n \rightarrow \infty}\left(k_{n}, l_{n}\right) \cdot \bar{a}_{t}=\infty$. 
We say that an orbit of $\mathbf{x} \in \mathscr{M}\left(\bar{a}_{1}, \ldots, \bar{a}_{t}\right)$ under $\bar{\Gamma}_{t}$-action $\left\{\tau_{k, l} \mathbf{x} \mid(k, l) \in \bar{\Gamma}_{t}\right\}$ is a heteroclinic orbit if $\tau_{k, l} \mathbf{x} \rightarrow \mathbf{x}^{-}$if $(k, l) \cdot \bar{a}_{t} \rightarrow-\infty$ and $\tau_{k, l} \mathbf{x} \rightarrow \mathbf{x}^{+}$if $(k, l) \cdot \bar{a}_{t} \rightarrow+\infty$. In [15], $\mathbf{x}^{-}, \mathbf{x}^{+}$have the following properties:

(i) $\mathbf{x}^{-}$and $\mathbf{x}^{+}$are more periodic than $\mathbf{x}$ since $\tau_{k, l} \mathbf{x}^{ \pm}=\mathbf{x}^{ \pm}$for all $(k, l) \in \bar{\Gamma}_{t}$, while $\mathbf{x}$ does not have such a property.

(ii) $\mathbf{x}^{-}$and $\mathbf{x}^{+}$are neighboring elements in $\mathscr{M}\left(\bar{a}_{1}, \ldots, \bar{a}_{t-1}\right)$, that is, there are no minimizers in $\mathscr{M}\left(\bar{a}_{1}, \ldots, \bar{a}_{t-1}\right)$ lying between $\mathbf{x}^{-}$and $\mathbf{x}^{+}$.

In the following, we want to prove that there exist neighboring elements in $\mathscr{M}\left(\bar{a}_{1}, \ldots\right.$, $\left.\bar{a}_{t-1}\right)$, i.e., there exist gaps in $\mathscr{M}\left(\bar{a}_{1}, \ldots, \bar{a}_{t-1}\right)$.

\section{The existence of gaps in $\mathscr{M}\left(\bar{a}_{1}, \ldots, \bar{a}_{t-1}\right)$}

In this section, we discuss the solutions of equation (1.3) for $0<\varepsilon \ll 1$. Using the antiintegrable limit method, an example of nonexistent minimal foliations will be constructed. The following lemmas are similar to the results in reference [11].

Let $R_{i}(\mathbf{x})=\sum_{j \in \mathbb{Z}^{d}} \partial_{i} h_{j}(\mathbf{x})$, then (1.3) becomes

$$
V^{\prime}\left(x_{i}\right)+\varepsilon R_{i}(\mathbf{x})=0, \quad i \in \mathbb{Z}^{d}, \text { and } \mathbf{x}=\left(x_{i}\right)_{i \in \mathbb{Z}^{d}} \in \mathbb{R}^{\mathbb{Z}^{d}} .
$$

By the definition of $h_{j}, R_{i}(\mathbf{x})=\sum_{j \in \mathbb{Z}^{d}} \partial_{i} h_{j}(\mathbf{x})=\sum_{\|j-i\| \leq r} \partial_{i} h_{j}(\mathbf{x})$.

If $\varepsilon=0$, then

$$
V^{\prime}\left(x_{i}\right)=0, \quad i \in \mathbb{Z}^{d} .
$$

Let $\|\mathbf{x}\|_{\infty}:=\sup _{i \in \mathbb{Z}^{d}}\left|x_{i}\right|$ and $B_{\delta}(\mathbf{x}):=\left\{\mathbf{X} \in \mathbb{R}^{\mathbb{Z}^{d}} \mid\|\mathbf{X}-\mathbf{x}\|_{\infty} \leq \delta\right\}$.

Lemma 3.1 Assume that $\mathbf{x}: \mathbb{Z}^{d} \rightarrow \mathbb{R}$ is a solution of (3.2) with the property that

$$
\operatorname{osc}(\mathbf{x}):=\sup _{\|j-i\| \leq r}\left|x_{j}-x_{i}\right| \leq K<\infty .
$$

Then there exist $\varepsilon_{0}>0$ and $\delta_{0}>0$, depending only on $K$, such that for every $\varepsilon \in\left[0, \varepsilon_{0}\right]$ there is unique $\mathbf{x}(\varepsilon) \in B_{\delta_{0}}(\mathbf{x})$ that satisfies (3.1). Moreover, $\lim _{\varepsilon \rightarrow 0}\|\mathbf{x}(\varepsilon)-\mathbf{x}\|_{\infty}=0$.

Proof First, by the continuity of $\partial_{i} h_{j}$ and assumption (H1), there exists a uniform constant $C_{1}>0$ for which

$$
\left|\partial_{i} h_{j}(\mathbf{X})\right| \leq C_{1} /(2 r+1)^{d} \quad \text { for all } i, j \in \mathbb{Z}^{d} \text { and all } \mathbf{X} \text { with } \operatorname{osc}(\mathbf{X}) \leq K+1 \text {. }
$$

Then $\left|R_{i}(\mathbf{X})\right| \leq \sum_{\|j-i\| \leq r}\left|\partial_{i} h_{j}(\mathbf{X})\right| \leq C_{1}$ for every $\mathbf{X}$ with osc $(\mathbf{X}) \leq K+1$. Particularly, if $\operatorname{osc}(\mathbf{x}) \leq K$ and $\|\mathbf{X}-\mathbf{x}\|_{\infty} \leq \frac{1}{2}$, then

$$
\operatorname{osc}(\mathbf{X}) \leq \operatorname{osc}(\mathbf{x})+\operatorname{osc}(\mathbf{X}-\mathbf{x}) \leq \operatorname{osc}(\mathbf{x})+2\|\mathbf{X}-\mathbf{x}\|_{\infty} \leq K+1
$$

Hence $\left|R_{i}(\mathbf{X})\right| \leq C_{1}$.

Because $V$ is a Morse function, there exists a constant $c>0$ such that $\left|V^{\prime \prime}\left(x_{i}\right)\right| \geq \frac{1}{c}$ for all $i \in \mathbb{Z}^{d}$. 
Let $\mathcal{X}=\left\{\mathbf{X} \in \mathbb{R}^{\mathbb{Z}^{d}} \mid\|\mathbf{X}-\mathbf{x}\|_{\infty}<\infty\right\}$. Define the operator $K_{\varepsilon, \mathbf{x}}: B_{\frac{1}{2}}(\mathbf{x}) \rightarrow \mathcal{X}$ by

$$
K_{\varepsilon, \mathbf{x}}(\mathbf{X})_{i}=X_{i}-\frac{V^{\prime}\left(X_{i}\right)+\varepsilon R_{i}(\mathbf{X})}{V^{\prime \prime}\left(x_{i}\right)}, \quad i \in \mathbb{Z}^{d}
$$

As observed above, $K_{\varepsilon, \mathbf{x}}$ is well defined on $B_{\frac{1}{2}}(\mathbf{x})$, and it is clear that $\mathbf{X}$ is a solution of (3.1) if and only if $K_{\varepsilon, \mathbf{x}}(\mathbf{X})=\mathbf{X}$, i.e., $\mathbf{X}$ is a fixed point of $K_{\varepsilon, \mathbf{x}}$.

Next, we take $0<k<1$ and select the appropriate $\varepsilon_{0}, \delta_{0}$ such that $K_{\varepsilon, \mathbf{x}}$ becomes a contracting operator with a contracting coefficient of $k$. Because $V$ only has finitely distinct critical points on $[0,1]$, sufficiently small $\delta_{0}$ (may let $0<\delta_{0}<\frac{1}{2}$ ) can be selected to make $\left\{\left(\zeta_{i}-\delta_{0}, \zeta_{i}+\delta_{0}\right) \mid \zeta_{i}\right.$ are different critical points of $\left.V\right\}$ disjoint. Furthermore, due to the continuity of $V^{\prime \prime},\left|V^{\prime \prime}\left(X_{i}\right)-V^{\prime \prime}\left(x_{i}\right)\right| \leq \frac{k}{2 c}$ uniformly for $\mathbf{X} \in B_{\delta_{0}}(\mathbf{x})$.

For any $\mathbf{y}, \mathbf{z} \in B_{\delta_{0}}(\mathbf{x})$, we have

$$
\left|K_{\varepsilon, \mathbf{x}}(\mathbf{y})_{i}-K_{\varepsilon, \mathbf{x}}(\mathbf{z})_{i}\right| \leq\left|\left(y_{i}-z_{i}\right)-\frac{V^{\prime}\left(y_{i}\right)-V^{\prime}\left(z_{i}\right)}{V^{\prime \prime}\left(x_{i}\right)}\right|+\varepsilon\left|\frac{R_{i}(\mathbf{y})-R_{i}(\mathbf{z})}{V^{\prime \prime}\left(x_{i}\right)}\right|
$$

We estimate the first item on the right-hand side:

$$
\begin{aligned}
\left|\left(y_{i}-z_{i}\right)-\frac{V^{\prime}\left(y_{i}\right)-V^{\prime}\left(z_{i}\right)}{V^{\prime \prime}\left(x_{i}\right)}\right| & =\left|\frac{\left(y_{i}-z_{i}\right)}{V^{\prime \prime}\left(x_{i}\right)}\left(\int_{0}^{1}\left[V^{\prime \prime}\left(x_{i}\right)-V^{\prime \prime}\left(t y_{i}+(1-t) z_{i}\right)\right] d t\right)\right| \\
& \leq \frac{k}{2}\|\mathbf{y}-\mathbf{z}\|_{\infty} .
\end{aligned}
$$

This shows that the first item is bounded by $\frac{k}{2}\|\mathbf{y}-\mathbf{z}\|_{\infty}$.

Then we estimate the second item on the right-hand side. Similar to the discussion above, we can see that $\partial_{i, l} h_{j}(\mathbf{X})$ is uniformly bounded on $B_{\frac{1}{2}}(\mathbf{x})(\Rightarrow \operatorname{osc}(\mathbf{X}) \leq K+1)$. Thus there exists $C_{2}>0$ such that

$$
\left|\partial_{i, l} h_{j}(\mathbf{X})\right| \leq C_{2} /(2 r+1)^{2 d}
$$

Hence,

$$
\begin{aligned}
\varepsilon\left|\frac{R_{i}(\mathbf{y})-R_{i}(\mathbf{z})}{V^{\prime \prime}\left(x_{i}\right)}\right| & \leq c \varepsilon \sum_{\|j-i\| \leq r}\left|\partial_{i} h_{j}(\mathbf{y})-\partial_{i} h_{j}(\mathbf{z})\right| \\
& =c \varepsilon \sum_{\|j-i\| \leq r}\left|\int_{0}^{1} \frac{d}{d t} \partial_{i} h_{j}(t \mathbf{y}+(1-t) \mathbf{z}) d t\right| \\
& \leq c \varepsilon \sum_{\|j-i\| \leq r} \sum_{\|l-j\| \leq r} \int_{0}^{1}\left|\partial_{i, l} h_{j}(t \mathbf{y}+(1-t) \mathbf{z})\right| d t \cdot\left|y_{l}-z_{l}\right| \\
& \leq c \varepsilon C_{2}\|\mathbf{y}-\mathbf{z}\|_{\infty} \\
& \leq \frac{k}{2}\|\mathbf{y}-\mathbf{z}\|_{\infty}\left(\varepsilon \leq \frac{k}{2 c C_{2}}\right) .
\end{aligned}
$$

Summarizing, we found that

$$
\left\|K_{\varepsilon, \mathbf{x}}(\mathbf{y})-K_{\varepsilon, \mathbf{x}}(\mathbf{z})\right\|_{\infty} \leq k\|\mathbf{y}-\mathbf{z}\|_{\infty} .
$$


Then we investigate whether $K_{\varepsilon, \mathbf{x}}$ maps $B_{\delta_{0}}(\mathbf{x})$ to $B_{\delta_{0}}(\mathbf{x})$. For any $\mathbf{y} \in B_{\delta_{0}}(\mathbf{x})$ and $i \in \mathbb{Z}^{d}$,

$$
\begin{aligned}
\left|K_{\varepsilon, \mathbf{x}}(\mathbf{y})_{i}-x_{i}\right| & \leq\left|K_{\varepsilon, \mathbf{x}}(\mathbf{y})_{i}-K_{\varepsilon, \mathbf{x}}(\mathbf{x})_{i}\right|+\left|K_{\varepsilon, \mathbf{x}}(\mathbf{x})_{i}-x_{i}\right| \\
& \leq k\|\mathbf{y}-\mathbf{x}\|_{\infty}+c C_{1} \varepsilon \\
& \leq k \delta_{0}+c C_{1} \varepsilon \\
& \leq \delta_{0}\left(\varepsilon \leq \frac{(1-k) \delta_{0}}{c C_{1}}\right) .
\end{aligned}
$$

It suffices to choose $0 \leq \varepsilon \leq \varepsilon_{0}:=\min \left\{\frac{(1-k) \delta_{0}}{c C_{1}}, \frac{k}{2 c C_{2}}\right\}$ to make sure that $K_{\varepsilon, \mathbf{x}}$ is a contracting operator from $B_{\delta_{0}}(\mathbf{x})$ to $B_{\delta_{0}}(\mathbf{x})$. Thus, there exists a unique fixed point $\mathbf{x}(\varepsilon) \in B_{\delta_{0}}(\mathbf{x})$ which is the solution of (3.1).

Finally, we prove that $\lim _{\varepsilon \rightarrow 0}\|\mathbf{x}(\varepsilon)-\mathbf{x}\|_{\infty}=0$. Since

$$
\begin{aligned}
\|\mathbf{x}(\varepsilon)-\mathbf{x}\|_{\infty} & =\left\|K_{\varepsilon, \mathbf{x}}(\mathbf{x}(\varepsilon))-K_{\varepsilon, \mathbf{x}}(\mathbf{x})+K_{\varepsilon, \mathbf{x}}(\mathbf{x})-\mathbf{x}\right\|_{\infty} \\
& \leq\left\|K_{\varepsilon, \mathbf{x}}(\mathbf{x}(\varepsilon))-K_{\varepsilon, \mathbf{x}}(\mathbf{x})\right\|_{\infty}+\left\|K_{\varepsilon, \mathbf{x}}(\mathbf{x})-\mathbf{x}\right\|_{\infty} \\
& \leq k\|\mathbf{x}(\varepsilon)-\mathbf{x}\|_{\infty}+c C_{1} \varepsilon .
\end{aligned}
$$

We get $\|\mathbf{x}(\varepsilon)-\mathbf{x}\|_{\infty} \leq \frac{c C_{1} \varepsilon}{1-k}$.

This lemma is similar to Theorem 3.1 in reference [11]. To understand the following content better, the proof is written out again.

In the following, let $\Gamma$ denote the set of solutions to (3.2) with the property that osc $(\mathbf{x}) \leq$ $K<\infty$, i.e.,

$$
\Gamma=\left\{\mathbf{x} \in \mathbb{R}^{\mathbb{Z}^{d}} \mid \mathbf{x} \text { is the solution of (3.2) and } \operatorname{osc}(\mathbf{x}) \leq K<\infty\right\}
$$

For every $\mathbf{x} \in \Gamma$, solution $\mathbf{x}(\varepsilon)$ of (3.1) can be obtained from Lemma 3.1. Use $\Gamma_{\varepsilon}$ to represent the collection of these $\mathbf{x}(\varepsilon)$, i.e.,

$$
\Gamma_{\varepsilon}=\{\mathbf{x}(\varepsilon) \mid \mathbf{x} \in \Gamma\}, \quad 0 \leq \varepsilon \leq \varepsilon_{0} .
$$

Lemma 3.2 $\Gamma$ and $\Gamma_{\varepsilon}$ are defined above. Then the map

$$
\Phi_{\varepsilon}: \Gamma \rightarrow \Gamma_{\varepsilon}, \quad \mathbf{x} \mapsto \mathbf{x}(\varepsilon)
$$

is a homeomorphism in the topology of pointwise convergence.

The proof is similar to Theorem 3.3 in reference [11]. It is omitted here.

Lemma 3.3 Assume $\omega \in \mathbb{R}^{d}$. Let $K>0$ be such that $\operatorname{osc}(\mathbf{x}) \leq K$ for all $\mathbf{x} \in \mathcal{B}_{\omega}$, and let $\varepsilon_{0}, \delta_{0}$ be as in Lemma 3.1. Then there is an $\varepsilon_{1}\left(0<\varepsilon_{1} \leq \varepsilon_{0}\right)$ such that, for all $0 \leq \varepsilon \leq \varepsilon_{1}$, the following is true:

When $\mathbf{x}^{\varepsilon}$ is any Birkhoff solution to (3.1) of rotation vector $\omega$, then $\mathbf{x}^{\varepsilon} \in B_{\delta_{0}}(\mathbf{x})$ for some Birkhoff solution $\mathbf{x}$ of (3.2) and hence $\mathbf{x}^{\varepsilon}=\mathbf{x}(\varepsilon)$. 
The proof can be found in Theorem 5.4 of reference [11]. It is also omitted here.

This lemma shows that the Birkhoff solution of (3.1) must be extended from the solution of (3.2) according to Lemma 3.1.

Definition 3.4 A nonempty set $\mathscr{F}$ consisting of stationary points is said to be a foliation, if

(1) $\mathscr{F}$ is a closed set under the product topology;

(2) $\mathscr{F}$ is totally ordered;

(3) $\mathscr{F}$ is invariant for translations;

(4) $p_{0}(\mathscr{F})=\mathbb{R}$.

Lemma 3.5 Let $\left(\bar{a}_{1}, \ldots, \bar{a}_{t}\right)$ be an admissible system. The set $\mathscr{M}\left(\bar{a}_{1}\right) \cup \cdots \cup \mathscr{M}\left(\bar{a}_{1}, \ldots, \bar{a}_{t}\right)$ is a foliation if and only if $\mathscr{M}\left(\bar{a}_{1}, \ldots, \bar{a}_{t}\right)$ does not have gaps.

Proof First, we assume that $\mathscr{M}\left(\bar{a}_{1}, \ldots, \bar{a}_{t}\right)$ does not have gaps. Next, it is proved that the set $\mathscr{M}\left(\bar{a}_{1}\right) \cup \cdots \cup \mathscr{M}\left(\bar{a}_{1}, \ldots, \bar{a}_{t}\right)$ is a foliation.

Because $\mathscr{M}\left(\bar{a}_{1}\right) \cup \cdots \cup \mathscr{M}\left(\bar{a}_{1}, \ldots, \bar{a}_{t}\right)$ is totally ordered (see Theorem 9.7 in [15]), we only have to show that the image of mapping $H$ satisfies $\operatorname{Im}(H)=\mathbb{R}$, where $H$ is defined as follows:

$$
H: \mathscr{M}\left(\bar{a}_{1}\right) \cup \cdots \cup \mathscr{M}\left(\bar{a}_{1}, \ldots, \bar{a}_{t}\right) \rightarrow \mathbb{R}, \quad \mathbf{x} \mapsto x_{0} .
$$

It is also known from Theorem 9.7 in [15] that $\mathscr{M}\left(\bar{a}_{1}\right) \cup \cdots \cup \mathscr{M}\left(\bar{a}_{1}, \ldots, \bar{a}_{t}\right)$ is closed. So $\operatorname{Im}(H)$ is closed. In fact, if $\mathbf{x}^{n} \in \mathscr{M}\left(\bar{a}_{1}\right) \cup \ldots \cup \mathscr{M}\left(\bar{a}_{1}, \ldots, \bar{a}_{t}\right)$ and $x_{0}^{n} \rightarrow x^{*}$ as $n \rightarrow \infty$. Let us prove $x^{*} \in \operatorname{Im}(\mathrm{H})$. Because $\mathscr{M}\left(\bar{a}_{1}\right) \cup \cdots \cup \mathscr{M}\left(\bar{a}_{1}, \ldots, \bar{a}_{t}\right) \subseteq \mathscr{M}_{\omega}(\omega$ is the rotation vector $)$ and $x_{0}^{n}$ is bounded, we know that $\left\{\mathbf{x}^{n}\right\}$ contains a subsequence converging to a minimizer $\tilde{\mathbf{x}}$ by the property $\left|x_{j}^{n}-x_{0}^{n}-j \cdot \omega\right| \leq 1$ and Tychonoff's theorem in the product topology. Since $\mathscr{M}\left(\bar{a}_{1}\right) \cup \cdots \cup \mathscr{M}\left(\bar{a}_{1}, \ldots, \bar{a}_{t}\right)$ is a closed set, $\tilde{\mathbf{x}} \in \mathscr{M}\left(\bar{a}_{1}\right) \cup \cdots \cup \mathscr{M}\left(\bar{a}_{1}, \ldots, \bar{a}_{t}\right)$ and $\tilde{x}_{0}=x^{*}$. So $x^{*} \in \operatorname{Im}(\mathrm{H})$. Hence, $\operatorname{Im}(\mathrm{H})$ is a closed set. $\operatorname{If} \operatorname{Im}(H) \neq \mathbb{R}$, we can find $x_{0}<y_{0} \in \operatorname{Im}(H)$ and $\left(x_{0}, y_{0}\right) \cap \operatorname{Im}(H)=\emptyset$.

This shows that $\bar{\Gamma}^{+}(\mathbf{x})=\bar{\Gamma}^{+}(\mathbf{y})$, where $\bar{\Gamma}^{+}(\mathbf{x})=\left\{\bar{k} \in \mathbb{Z}^{d+1} \mid \tau_{\bar{k}} \mathbf{x} \geq \mathbf{x}\right\}$. Since $\bar{\Gamma}^{+}(\mathbf{x})=\bar{\Gamma}^{+}(\mathbf{y}), \mathbf{x}$ and $\mathbf{y}$ have the same secondary invariants. Then there exists $s(1 \leq s \leq t)$ such that $\mathbf{x}, \mathbf{y}$ are neighboring elements in $\mathscr{M}\left(\bar{a}_{1}, \ldots, \bar{a}_{s}\right)$.

When $s=t$, this contradicts nonexistence of gaps in $\mathscr{M}\left(\bar{a}_{1}, \ldots, \bar{a}_{t}\right)$.

When $s<t$, by Theorem 2.3 , there exists $\mathbf{w} \in \mathscr{M}\left(\bar{a}_{1}, \ldots, \bar{a}_{s+1}\right)$ with $\mathbf{x} \ll \mathbf{w} \ll \mathbf{y}$. This contradicts $\left(x_{0}, y_{0}\right) \cap \operatorname{Im}(H)=\emptyset$.

Then we assume that the set $\mathscr{M}\left(\bar{a}_{1}\right) \cup \cdots \cup \mathscr{M}\left(\bar{a}_{1}, \ldots, \bar{a}_{t}\right)$ is a foliation. Next we prove that $\mathscr{M}\left(\bar{a}_{1}, \ldots, \bar{a}_{t}\right)$ does not have gaps.

If there are gaps in $\mathscr{M}\left(\bar{a}_{1}, \ldots, \bar{a}_{t}\right)$ and $\mathbf{x}^{1} \ll \mathbf{x}^{2}$ is one of them, then there is $\mathbf{x} \in \mathscr{M}$ which satisfies $\mathbf{x}^{1} \ll \mathbf{x} \ll \mathbf{x}^{2}$ and $t(\mathbf{x})>t$. This contradicts the assumption that the set $\mathscr{M}\left(\bar{a}_{1}\right) \cup$ $\cdots \cup \mathscr{M}\left(\bar{a}_{1}, \ldots, \bar{a}_{t}\right)$ is a foliation.

Theorem A Assume that $\left(\bar{a}_{1}, \ldots, \bar{a}_{t}\right)$ is an admissible system. $\varepsilon$ is defined as Lemma 3.3, then the minimal solutions set $\mathscr{M}\left(\bar{a}_{1}\right) \cup \ldots \cup \mathscr{M}\left(\bar{a}_{1}, \ldots, \bar{a}_{t-1}\right)$ corresponding to the local potential $h_{j}^{\varepsilon}\left(\right.$ or $\left.W^{\varepsilon}\right)$ is not a foliation.That is to say, there are gaps in $\mathscr{M}\left(\bar{a}_{1}, \ldots, \bar{a}_{t-1}\right)$, so $\mathscr{M}\left(\bar{a}_{1}, \ldots, \bar{a}_{t}\right) \neq \emptyset$. 
Proof We prove it by contradiction. If the set $\mathscr{M}\left(\bar{a}_{1}\right) \cup \cdots \cup \mathscr{M}\left(\bar{a}_{1}, \ldots, \bar{a}_{t-1}\right)$ is a foliation, we denote the foliation by $\mathscr{F}$. By Lemma 3.3, $\mathscr{F} \subset\left(\Gamma_{\varepsilon} \cap \mathcal{B}\right)$. Then, by Lemma 3.2, $\Phi_{\varepsilon}^{-1}(\mathscr{F}) \subset \Gamma$.

Because $\mathscr{F}$ is connected, under continuous mapping, its image $\Phi_{\varepsilon}^{-1}(\mathscr{F})$ is also connected. But $\Gamma$ is a completely disconnected set. This is a contradiction. In fact, $\Gamma$ satisfies the first countable axiom (each point has a countable neighborhood base), so the finite set is a closed set and the special single point set is also a closed set. Because the critical point of Morse function is isolated, the single point set in $\Gamma$ is an open set. Thus, the single point set in $\Gamma$ is both open and closed, $\Gamma$ is completely disconnected.

We recall that if $\left(\bar{a}_{1}, \ldots, \bar{a}_{t}\right)$ is admissible, then $\left(\bar{a}_{1}, \ldots, \bar{a}_{t-1}\right)$ is admissible. By Lemma 3.5, there are gaps in $\mathscr{M}\left(\bar{a}_{1}, \ldots, \bar{a}_{t-1}\right)$. Then, by Theorem $2.3, \mathscr{M}\left(\bar{a}_{1}, \ldots, \bar{a}_{t}\right) \neq \emptyset$.

Therefore, under the condition of Theorem A, we find an example of the nonexistence of the minimal foliation. Thus, the hypothesis of Theorem 2.3 can be established, and the heteroclinic orbit can be determined.

\author{
Acknowledgements \\ Not applicable. \\ Funding \\ National Natural Science Foundation of China (11701298).
}

Availability of data and materials

Not applicable.

Competing interests

The author declares that they have no competing interests.

Author's contributions

XQM wrote the manuscript alone. She read and approved the final manuscript.

\title{
Authors' information
}

Author: Xue-Qing Miao. My research focuses on the Frenkel-Kontorova model and its applications. Relevant research results have been published in J. Differential Equations, Advances in Mathematics, Ergodic Theory Dynam. Systems, and Int. J. Bifur. Chaos.

\section{Publisher's Note}

Springer Nature remains neutral with regard to jurisdictional claims in published maps and institutional affiliations.

Received: 23 May 2020 Accepted: 16 December 2020 Published online: 28 January 2021

References

1. Aubry, S., Le Daeron, P.Y.: The discrete Frenkel-Kontorova model and its extensions. Physica D 8, 381-422 (1983)

2. Bangert, V:: A uniqueness theorem for $\mathbb{Z}^{n}$-periodic variational problems. Comment. Math. Helv. 62, $511-531$ (1987)

3. Bangert, $\vee$ :: The existence of gaps in minimal foliations. Aequ. Math. 34, 153-166 (1987)

4. Bangert, V.: Mather sets for twist maps and geodesics on tori. In: Kirchgraber, U., Walther, H.O. (eds.) Dynamics Reported, vol. 1, pp. 1-56. Wiley, New York (1988)

5. Bangert, V:: On minimal laminations of the torus. Ann. Inst. Henri Poincaré, Anal. Non Linéaire 6, 95-138 (1989)

6. Bessi, U.: Many solutions of elliptic problems on $\mathbb{R}^{n}$ of irrational slope. Commun. Partial Differ. Equ. 30, 1773-1804 (2005)

7. Blank, M.L.: Metric properties of minimal solutions of discrete periodical variational problems. Nonlinearity $2,1-22$ (1989)

8. de la Llave, R., Valdinoci, E.: Ground states and critical points for generalized Frenkel-Kontorova models in $\mathbb{Z}^{d}$. Nonlinearity 20, 2409-2424 (2007)

9. de la Llave, R., Valdinoci, E.: A generalization of Aubry-Mather theory to partial differential equations and pseudo-differential equations. Ann. Inst. Henri Poincaré, Anal. Non Linéaire 26, 1309-1344 (2009)

10. de la Llave, R., Valdinoci, E.: Ground states and critical points for Aubry-Mather theory in statistical mechanics. J. Nonlinear Sci. 20, 153-218 (2010)

11. Knibbeler, V., Mramor, B., Rink, B.: The laminations of a crystal near an anti-continuum limit. Nonlinearity 27, 927-952 (2014)

12. Koch, H., de la Llave, R., Radin, C.: Aubry-Mather theory for functions on lattices. Discrete Contin. Dyn. Syst., Ser. A 3 , 135-151 (1997) 
13. Li, W.-L., Cui, X.-J.: Heteroclinic solutions for a generalized Frenkel-Kontorova model by minimization methods of Rabinowitz and Stredulinsky. J. Differ. Equ. 268, 1106-1155 (2020)

14. Mather, J.N.: Existence of quasi-periodic orbits for twist homeomorphisms of the annulus. Topology 21, 457-467 (1982)

15. Miao, X.-Q., Qin, W.-X., Wang, Y.-N.: Secondary invariants of Birkhoff minimizers and heteroclinic orbits. J. Differ. Equ. 260, 1522-1557 (2016)

16. Moser, J.: Minimal solutions of variational problems on a torus. Ann. Inst. Henri Poincaré, Anal. Non Linéaire 3 , 229-272 (1986)

17. Mramor, B., Rink, B.: Ghost circles in lattice Aubry-Mather theory. J. Differ. Equ. 252, 3163-3208 (2012)

18. Mramor, B., Rink, B.: A dichotomy theorem for minimizers of monotone recurrence relations. Ergod. Theory Dyn. Syst. 35, 215-248 (2015)

19. Su, X., de la Llave, R.: Percival Lagrangian approach to the Aubry-Mather theory. Expo. Math. 30, 182-208 (2012)

Submit your manuscript to a SpringerOpen ${ }^{\circ}$ journal and benefit from:

- Convenient online submission

- Rigorous peer review

- Open access: articles freely available online

- High visibility within the field

- Retaining the copyright to your article

Submit your next manuscript at $\gg$ springeropen.com 